\title{
Correlation, Independance and Inverse Modeling
}

\author{
V. Vigneron ${ }^{1,2}$ \\ ${ }^{1}$ IBISC-lab CNRS FRE 3190 \\ Université d'Evry, 91020 Evry Cedex, France \\ vincent.vigneron@ibisc .univ-evry.fr \\ 2 Équipe MATISSE-SAMOS CES CNRS-UMR 8173 \\ 75634 Paris cedex 13,France \\ vigneron@univ-paris1.fr
}

\begin{abstract}
Learning from examples has a wide number of forms depending on what is to be learned from which available information. One of these form is $\mathbf{y}=f(\mathbf{x})$ where the input-output pair $(\mathbf{x}, \mathbf{y})$ is the available information and $f$ represents the process mapping $\mathbf{x} \in X$ to $\mathbf{y} \in \mathcal{Y}$. In general and for real world problems, it is not reasonnable to expect having the exact representation of $f$. A fortiori when the dimension of $\mathbf{x}$ is large and the number of examples is little. In this paper, we introduce a new model, capable to reduce the complexity of many ill-posed problems without loss of generality. The underlying Bayesian artifice is presented as an alternative to the currently used frequency approaches which does not offer a compelling criterion in the case of high dimensional problems.
\end{abstract}

\section{Introduction}

Inverse problems are defined, as their name points it out as the opposite of direct problems. Such definition is empty of sense as long as one do not define what is a direct problem. An inverse problem is a situation in which one try to determine reasons of a phenomenon from the experimental observations of his effects. For instance, in seismology, the location of the origin of an earthquake from measurements made by several seismic stations is an inverse problem. The resolution of such problem goes through an initial stage of modeling of the phenomenon, which describes the influence of the parameters of the model translated experimentally in visible effects. Then, these parameters are estimated. Mathematical resolution is made difficult due to the fact that inverse problems are in general ill-posed problems, i. e the only observations are not enough to determine perfectly all parameters of the model. It is therefore necessary to add priors which allow to reduce the space of solutions in order to lead to an unique resolution. If a problem is well-posed, there are good chances that a resolution is found by a stable algorithm, on a computer. Otherwise, it must be reformulated for numerical treatments. Typically, it assumes additional hypotheses, for instance the regularity of the solution. This process is known under the name of regularization [7].

The mathematical term of "well-posed problem" comes from a definition of Hadamard. He thought that the mathematical models of physical phenomena should have the following properties 
1. one solution exists

2. the solution is single

3. resolution depends continuously on data, for a reasonable topology.

When several conclusions are consistent with the same data, the problem is to choose the best one, such as the one with minimum variance by example 3]. Although in terms of functional analysis, ill-posed problems are typically continuous, they may be unstable when they are solved with a finite precision, or with errors in the data.

\section{General Inverse Model}

In large scale problems (with a large number of sensors), the most common option is a reduction of dimensionality, usually performed in conjunction with a whitening step. Another approach, especially popular in the source separation community, is to work by deflation. This trail is usually followed for problems in which the number of sensors and the number of sources are both high [2]. The inherent problem with large dimension is the problem of empty space and the curse to dimensionality: observations quickly become very small samples in large sizes and are also noisy. The reduction is essential, but the methods are generally not robust w.r.t. noise and only applicable in a linear framework. If it is restricted to the linear framework, then one can not probably avoid taking into account the noise [4].

In source separation for instance, deflation techniques accumulate errors over time as the number of sources extracted increases [2]. The problem is important for many applications: EEG (electro-encephalography), BSPM (body surface potential mapping), MEG (magneto-encephalography), NMR (nuclear magnetic resonance), hyperspectral imaging, data analysis, etc.

Another way consists on the contrary to take advantage of the dimensionality of the data, as illustrated in Fig. 1. Take the example of a non-monotone relationship $x \rightarrow y=f(x)$. In figure 1 a. the solutions to the equation $y=f(x)$ at $y_{0}$ are $\left\{x_{1}, x_{2}, x_{3}\right\}$ and nothing can aid for distinguishing them. But, if several achievements of the relationship $y=f(x)$ are available (cf. Fig[1, b), these achievements can be combined in order to bring about a most likely solution than others. This example also highlights that "locality" supports the inverse approach.

When a mathematical problem is convex, it is in general quite easy to find the global minimum by using a local method. Hence the idea, in the non-convex case, is to partition the domain in order to approximate the non-convex problem on each subset of the partition, by a subset of convex problems. Most of non-convex problems can be approximated by a partition into convex problems, by "relaxing" certain constraints and the criterion [1]. A possible approach for choosing a relaxation strategy can be itemized into 4 stages :

- The choice of the structure: for one input vector $\mathbf{x}=\left(x_{1}, \ldots, x_{n}\right)^{T}$ of dimension $n$ and an output $\mathbf{y}$ of dimension $m$, there is $n$ "one-to-many" independent models $M_{i}, i=1, \ldots, n$, parameterized by a vector of parameters $\mathbf{p}_{i} \in \mathbb{R}^{\sigma(i)}$, where $\sigma(i)$ is the number of parameters for the model 


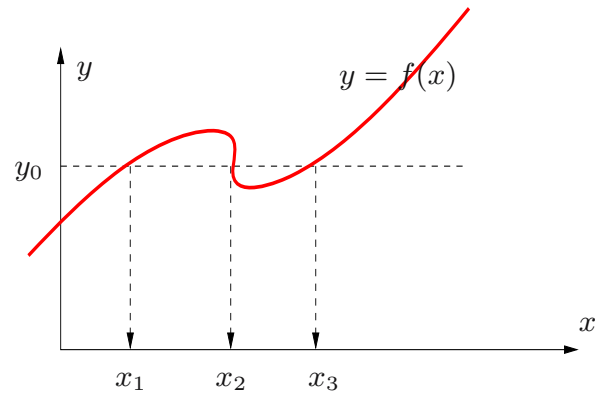

(a)

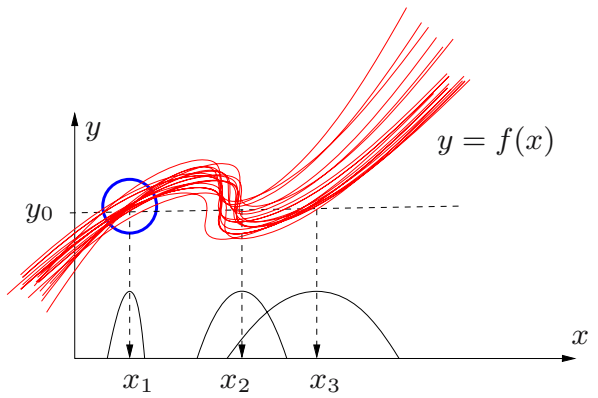

(b)

Fig. 1. (a) An instance of an ill-posed problem (b) $x_{0}$ is likely the solution sought because the density of the solutions is sharper around $x_{0}$ than around $x_{1}$ or $x_{3}$

$M_{i}$. One can have $\sigma(i)=\sigma(j), \forall i \neq j$, but not necessarily. In the following, $M(\mathbf{p})=\left\{M_{1}\left(\mathbf{p}_{1}\right), M_{2}\left(\mathbf{p}_{2}\right), \ldots, M_{n}\left(\mathbf{p}_{n}\right)\right\}$ denotes the structure of the model. Eligible prior set for the vector of parameters $\mathbf{p}=\left(\mathbf{p}_{1}, \ldots, \mathbf{p}_{n}\right)^{T}$ of the model is $\mathbb{R}^{\sigma}$, where $\sigma=\sum_{i} \sigma(i)$. Each parameter vector $\mathbf{p}_{i}$ is a model partner $M\left(\mathbf{p}_{i}\right)$ of the structure. The dependence between the output $x_{i}$ and the inputs $\mathbf{y}$ is linear (or not) w.r.t. the parameters $\mathbf{p}_{i}$.

- The data collection: vectors $\mathbf{x}$ and $\mathbf{y}$ are $N$ measurements collected on a multivariable system (multiple inputs and multiple outputs). Each model $M_{i}\left(\mathbf{y} ; \mathbf{p}_{i}\right)$ generates a scalar output $x_{M_{i}}\left(\mathbf{p}_{i}\right)$, homogeneous to the entry $x_{i}$. The error between the data $x_{i}$ and the model outpout $x_{M_{i}}\left(\mathbf{p}_{i}\right)$ is defined by:

$$
e\left(\mathbf{p}_{i}\right) \equiv e_{i}=x_{i}-x_{M_{i}}\left(\mathbf{p}_{i}\right) .
$$

- the estimation: it is to retain a model $M(\mathbf{p})$ generating outputs $x_{M_{1}}\left(\mathbf{p}_{1}\right), x_{M_{2}}\left(\mathbf{p}_{2}\right), \ldots, x_{M_{n}}\left(\mathbf{p}_{n}\right)$ sufficiently similar to the experimental data, i.e. such that error $e(\mathbf{p})$ be small in a sense to be defined.

- the inference: it aims to calculate the response of the model $\hat{\mathbf{y}}$ when the vector $\mathbf{x}$ feeds the model, combining the answers (and not vice versa). This combination of information is a sensitive thing that will be detailled in the Algorithm 1. The multi-model approach allows us to exploit models $M_{i}\left(\mathbf{p}_{i}\right)$ well-suited to the local complexity of the considered sub-problems and in which a structural rule $\phi(\cdot)$ is used to get $\hat{\mathbf{y}}$. How taking advantage of the information provided by the errors $\mathbf{e}$ to estimate $\hat{\mathbf{y}}$ ?

Suppose that the function $\mathbf{f}: \mathbb{R}^{m} \rightarrow \mathbb{R}^{n}$, associated to $M(\cdot)$, is continuous and locally injective. Note that the reverse function $\mathbf{f}^{-1}$ in general is not defined (even when $n=m$ ). However, it is never defined everywhere. It is difficult to study the type of discontinuity that may appear around $\mathbf{f}^{-1}$, but we will use the following definition:

Definition 1 (stability of a solution). The vector $\mathbf{y}$ defines a stable point if $\partial x_{M_{i}\left(\mathbf{y} ; \mathbf{p}_{i}\right)} / \partial \mathbf{y} \rightarrow 0$. 
When $\mathbf{y}$ is unstable, there is probably a compact containing $\mathbf{y}$ in which $\mathbf{f}^{-1}$ is discontinuous.

\section{Stochastic Search of the Solution}

Starting from the previous definition, we imagined an estimation procedure based on a Markov chain structure, which can be exploited to find the solution to the problem of inference.

The estimation procedure uses a MCMC approach that emphasizes the stochastic search to resolve the problem of inference. See [5] for more details. The Markov chain structure does not contain any attributed parameters. Let $\left\{\partial x_{M_{i}\left(\mathbf{y} ; \mathbf{p}_{i}\right)} / \partial y\right\}, i=1, \ldots, n$ be the jacobian values of the parcimonious models, the algorithm is the following one:

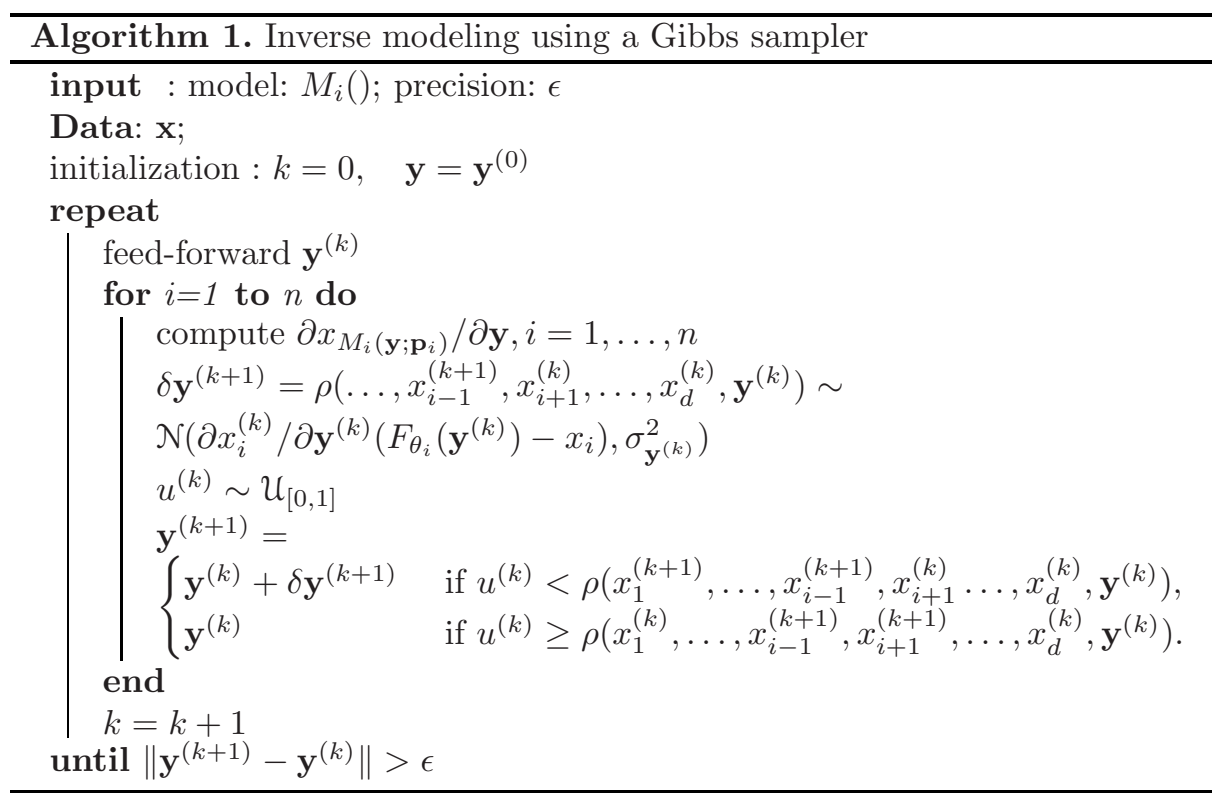

By setting $q\left(\mathbf{x}^{(k)}\right)=\exp \left(-\sum_{i=1}^{n}\left|\hat{x}_{M_{i}\left(\mathbf{y} ; \mathbf{p}_{i}\right)}^{(k)}-x_{i}^{(k)}\right|^{2} / 2 \sigma_{x}^{2}\right)$, Robert [5] propose to use the weighted estimation $\hat{\mathbf{y}}^{(k+1)}=\sum_{k} q\left(\mathbf{x}^{(k)}\right) \mathbf{y}^{(k)} / \sum_{k} q\left(\mathbf{x}^{(k)}\right)$ where $\sum_{k} q\left(\mathbf{x}^{(k)}\right)$ performs a normalisation of the coefficients $q\left(\mathbf{x}^{(k)}\right)$. The normal law $\mathcal{N}\left(\partial x_{i}^{(k)} / \partial \mathbf{y}^{(k)}, \sigma_{\mathbf{y}}^{2}\right)$ provides a probabilistic representation of the hazard in an hypercube centered on $J_{i}=\partial x_{M_{i}\left(\mathbf{y} ; \mathbf{p}_{i}\right)} / \partial y$ and of size $\left[-\sigma_{\mathbf{y}}^{2}, \sigma_{\mathbf{y}}^{2}\right]$. But, the random sampling stage $\delta\left(\mathbf{y}^{(k)}\right)$ can be encountered more frequently under the following form:

$$
\mathbf{y}^{(k+1)}=\left\{\begin{array}{ll}
\mathbf{y}^{(k)}+\delta \mathbf{y}^{(k+1)} & \text { if } u<\rho\left(\mathbf{p}^{(k)}, \mathbf{y}^{(k)}\right) \\
\mathbf{y}^{(k)} & \text { else }
\end{array} .\right.
$$


The sampling scheme proposed in (2) leads to no statistical bias. Robert [5] shown that the free random walk (constituted of these small jumps with no energetic constraint) is ergodic and therefore visits all sites in the model with the same frequency. Some remarks regarding the algorithm Tab. 1 it consists to test according to an energetic eligibility criterion a series of individual displacements $\left\{\delta \mathbf{y}^{(k)}\right\}_{t>1}$ obtained by drawing for each of them (2) all involved experts, according to the value of their dynamic variables $\partial x_{i} / \partial y, i=1, \ldots, d$. The displacement leads, from $\mathbf{y}^{(k)}$, to $\mathbf{y}^{(k+1)}$. It can be shown that the distribution of random configuration converge to the expected distribution $q\left(\mathbf{x}^{(k)}\right)$ if the number of simulations $T \rightarrow \infty$.

The Monte Carlo method makes it possible to circumvent the difficulties linked to the finite size of the model while preserving the statistical qualities of the simulation. One of the major difficulties is inevitably the bias due to the use of a discrete model to reproduce the real system.

The convergence properties in the chain $\left\{y^{(k)}\right\}$ are directly related to the Jacobien $J^{(k)}$ in the sense that $\left\{\mathbf{y}^{(k)}\right\}$ is aperiodic and irreducible.

\section{Convergence Properties}

Tarantola [6] and Tikhonov [7] have shown that it is not always possible to demonstrate the existence and uniqueness of the solution. To find the solution $\hat{\mathbf{y}}$ of the equation $f(\mathbf{y})=\mathbf{x}$, let us give a candidate Lyapunov function $V$ :

$$
V=\frac{1}{2}\|f(\mathbf{y})-\mathbf{x}\|^{2}, \mathbf{x} \in \mathbb{R}^{\mathbf{n}}, \mathbf{y} \in \mathbb{R}^{\mathbf{m}} .
$$

Let us note $J$ the $m \times n$ jacobian matrix defined by $\partial \mathbf{x} / \partial \mathbf{y}$ and the hessian matrix $\partial^{2} V / \partial x^{2}$. In the following, we will assume some additional conditions on the regularity of $J$ :

$$
\begin{aligned}
& V \in \mathcal{C}^{2}\left(\mathbb{R}^{p}\right) \text { is non-constant convex, } \\
& \lim _{|\mathbf{x}| \rightarrow \infty} V=+\infty
\end{aligned}
$$

$$
\begin{aligned}
& \left(\partial^{2} V / \partial \mathbf{y}^{2}\right) \text { is bounded and }\left\|\left(\partial^{2} V / \partial \mathbf{y}^{2}\right)\right\|_{\infty}=M . \\
& K=\sup _{\mathbf{x}}\left|\sum_{i=1}^{p}\left(\partial^{2} V / \partial \mathbf{y}^{2}\right)_{i i}(\mathbf{y})\right| .
\end{aligned}
$$

The fact that $V$ is non constant implies that $\|(\partial V / \partial \mathbf{y})(\mathbf{x})\|=\mathbf{0} \Leftrightarrow \mathbf{y}=\mathbf{y}^{*}$. The gradient of $V$ at the point $\mathbf{y}$ is $(\partial V / \partial \mathbf{y})$. Minimizing $V$ gives an adaption rule which guarantees convergence towards the solution of $\hat{\mathbf{x}}-f(\mathbf{y})=0$ in the sense of Lyapunov. This can be written

$$
\mathbf{y}^{(n+1)}=\mathbf{y}^{(n)}-\rho(\partial V / \partial \mathbf{y})^{T}\left(\mathbf{y}^{(n)}\right),
$$


with $\rho$ an adaptation factor. We can analyze in a specific way convergence properties of (7) by defining 2 other chains:

$$
p_{n}=\exp \left(-V\left(\mathbf{y}^{(n)}\right)\right) \quad \text { and } \quad \hat{\mathbf{y}}^{(n)}=\sum_{k=1}^{n} p_{k} \mathbf{y}^{(k)} / \sum_{k=1}^{n} p_{k}
$$

The definition of $\left(p_{k}\right)$ is intuitive and is designed to improve our estimation of $\mathbf{y}^{*}$. One could have chosen $p_{n}=\chi_{\mathcal{B}\left(\mathbf{x}^{*}, \delta\right)}\left(f\left(\mathbf{y}_{k}\right)\right)$, where $\mathcal{B}$ indicates the open ball, just to keep in the chain $\hat{\mathbf{y}}^{(n)}$ the values of $\mathbf{y}_{k}$ whose images by $f$ is distant from $\mathbf{x}^{*}$ of $<\delta$.

Lemma 1. The chain $E\left[V\left(\mathbf{y}^{(n)}\right)\right]$ is decreasing and converges toward 0 .

Proof (Lemma 1). This requires the second-order Taylor-Lagrange equality when $\mathbf{y}$ is in the vicinity of the optimum. There exists $\mathbf{z} \in\left[\mathbf{y}^{(n)}, \mathbf{y}^{(n+1)}\right]$ such that:

$$
\begin{aligned}
V\left(\mathbf{y}^{(n+1)}\right)-V\left(\mathbf{y}^{(n)}\right) & =\frac{\partial V^{T}}{\partial \mathbf{y}}\left(\mathbf{y}^{(n)}\right) \cdot\left(\mathbf{y}^{(n+1)}-\mathbf{y}^{(n)}\right)+\frac{1}{2}\left(\mathbf{y}^{(n+1)}-\mathbf{y}^{(n)}\right)^{T} \cdot \frac{\partial^{2} V}{\partial \mathbf{y}^{2}}(\mathbf{y}) \cdot\left(\mathbf{y}^{(n+1)}-\mathbf{y}^{(n)}\right) \\
& =-\rho(\partial V / \partial \mathbf{y})^{T}(\partial V / \partial \mathbf{y})+\frac{1}{2} \rho^{2}(\partial V / \partial \mathbf{y}) \cdot \frac{\partial^{2} V}{\partial \mathbf{y}^{2}}(\mathbf{y}) \cdot(\partial V / \partial \mathbf{y})^{T} .
\end{aligned}
$$

Taking the expectation, it comes:

$$
\begin{aligned}
E\left[V\left(\mathbf{y}^{(n+1)}\right)-V\left(\mathbf{y}^{(n)}\right)\right]= & -\rho(\partial V / \partial \mathbf{y})^{2}+ \\
& +\frac{1}{2} \rho^{2}(\partial V / \partial \mathbf{y})^{T} \cdot \frac{\partial^{2} V}{\partial \mathbf{y}^{2}}(\mathbf{y}) \cdot(\partial V / \partial \mathbf{y})+\frac{1}{2} \sum_{i=1}^{p}\left(\partial^{2} V / \partial \mathbf{y}^{2}\right)_{i i}(\partial V / \partial \mathbf{y})_{i}^{T} .
\end{aligned}
$$

From (5) and (6), it comes:

$$
E\left[V\left(\mathbf{y}^{(n+1)}\right)-V\left(\mathbf{y}^{(n)}\right)\right] \leq-\rho(\partial V / \partial \mathbf{y})^{2}+\frac{1}{2} \rho^{2} M(\partial V / \partial \mathbf{y})^{2}+\frac{1}{2} K \sigma^{2} .
$$

(9) is negative iif

$$
\rho \in\left[\frac{1}{M}-\frac{1}{M} \sqrt{1-\frac{K \sigma^{2}}{(\partial V / \partial \mathbf{y})^{2}}}, \frac{1}{M}+\frac{1}{M} \sqrt{1-\frac{K \sigma^{2}}{(\partial V / \partial \mathbf{y})^{2}}}\right] .
$$

In these circumstances, the chain $E\left(V\left(\mathbf{y}^{(n)}\right)\right)$ is decreasing and bounded, and thus converges.

Lemma 2. Let $f$ a fonction satisfying the conditions imposed on $V$, and $\left(\mathbf{x}_{n}\right)$ a chain in $\mathbb{R}^{p}$. For further simplifications, we suppose that $\mathbf{y}^{*}=0$. Then

$$
\lim _{n \rightarrow \infty} f\left(\mathbf{x}_{n}\right)=0 \Leftrightarrow \lim _{n \rightarrow \infty} \mathbf{x}_{n}=0 .
$$

Proof (Lemma Q Q The direction ' $\Leftarrow$ ' is evident because of the continuity of $f$. Let us reason by absurdity on the direction ' $\because$ '. Suppose that $\mathbf{x}_{n}$ does not go toward 0 . Then $\exists \epsilon, \forall N \in \mathbb{N}, \exists n>N /\left\|\mathbf{x}_{n}\right\|>\epsilon$. One could extract a chain $\left(u_{n}\right)$ 
from $\left(\mathbf{x}_{n}\right)$ such that $\forall n,\left\|u_{n}\right\|>\epsilon$. Consider now $m=\lim _{\mathbf{y} \in \mathbb{R}^{p} \backslash \mathcal{B}(0, \epsilon)} f(\mathbf{y})$. This lower limit exists as $f \geq 0$. In addition $m>0$ because (4) means that $f$ reaches its minimum on $\mathbb{R}^{p} \backslash \mathcal{B}(0, \epsilon)$ and $\mathbf{0}$ is the only point such that $f(\mathbf{0})=\mathbf{0}$. There is therefore a chain $f\left(u_{n}\right)$ extracted from $\left(\mathbf{x}_{n}\right)$ such that $\forall n, f\left(u_{n}\right)>m$ which is in contradiction with the hypothesis.

Theorem 1. The chain $E\left[\mathbf{y}^{(n)}\right]$ converges towards $\mathbf{y}^{*}$ when $n \rightarrow \infty$.

From Jensen inequality, since $V$ is convex $\forall n, V\left(E\left[\mathbf{y}^{(n)}\right]\right) \leq E\left[V\left(\mathbf{y}^{(n)}\right)\right]$. The result is obtained by applying the lemma (2) to the chain $E\left(\mathbf{y}^{(n)}\right)$.

We would like to show that the chain $E\left[\hat{\mathbf{y}}^{(n)}\right]$ converges toward $\mathbf{y}^{*}$ :

$$
E\left(\hat{\mathbf{y}}^{(n)}-\mathbf{y}^{*}\right)=\sum_{k=1}^{(n)} E\left[p_{k}\left(\mathbf{y}^{(k)}-\mathbf{y}^{*}\right) / \sum_{i=1}^{(n)} p_{i}\right]+\sum_{k=N+1}^{n} E\left[p_{k}\left(\mathbf{y}^{(k)}-\mathbf{y}^{*}\right) / \sum_{i=1}^{n} p_{i}\right]
$$

By applying Cauchy inequality:

$$
\left\|E\left[\hat{\mathbf{y}}^{(n)}-\mathbf{y}^{*}\right]\right\|^{2} \leq\left\|\sum_{k=1}^{n} E\left[p_{k}\left(\mathbf{y}^{(k)}-\mathbf{y}^{*}\right) / \sum_{i=1}^{n} p_{i}\right]\right\|^{2}+\sum_{k=N+1}^{n}\left\|E\left[\left(\mathbf{y}^{(k)}-\mathbf{y}^{*}\right)^{2}\right]\right\|\left\|E\left[\left(p_{k} / \sum_{i=1}^{n} p_{i}\right)^{2}\right]\right\| .
$$

An additional condition is necessary to demonstrate that $V\left(\mathbf{y}^{(k)}\right)$ converges towards $\mathbf{0}$. A second problem is to prove that $E\left[\left(p_{k} / \sum_{i=1}^{n} p_{i}\right)^{2}\right]$ tends toward $\mathbf{0}$, which is possible only if $V$ is radial.

The calculus of the weighted estimator $\hat{\mathbf{y}}^{(n)}=\sum_{k=1}^{n} p_{k} \mathbf{y}^{(k)} / \sum_{k=1}^{n} p_{k}$ (see Tab!) is appealing. But its use can lead to disastrous results if the number of simulations is insufficient (in practice $<1000$ according to Robert [5]). It is a condition for almost sure convergence $\hat{\mathbf{y}} \stackrel{a s}{\sim} E_{P}[\mathbf{y}]$.

It is easy to show that the convergence of the weighted estimator $\mathbf{y}^{(n)}$ is faster than the uniform estimator. Compare for instance $S_{n}=\sum_{k=1}^{n} \frac{1}{n}\left(\mathbf{y}^{(k)}-\mathbf{y}^{*}\right)^{2}$ and $\hat{S}_{n}=\sum_{k=1}^{n} p_{k} / \sum_{i=1}^{n} p_{i}\left(\mathbf{y}^{(k)}-\mathbf{y}^{*}\right)^{2}$. Consider the function $g(\mathbf{y})=p(\mathbf{y}) / \sum_{i=1}^{n} p_{i}$ where $p(\mathbf{y})=\exp (-V(\mathbf{y}))$ and $p_{i}=\exp \left(-V\left(\mathbf{y}_{i}\right)\right)$. Let us denote $q_{i}=g\left(\mathbf{y}_{i}\right) \cdot g$ is defined on $\mathbb{R}^{p}$ with values in $\mathbb{R}_{+}$. Since $\forall i, p_{i} \leq 1$, one has $\sum_{i=1}^{n} p_{i} \leq n$, hence:

$$
g\left(\mathbf{y}^{*}\right)=1 / \sum_{i=1}^{n} p_{i} \geq \frac{1}{n}
$$

Moreover $\lim _{|\mathbf{y}| \rightarrow \infty} g(\mathbf{y})=0$. So $\left\{\frac{1}{n}\right\} \in g\left(\mathbb{R}^{p}\right)$. Let $\mathcal{V}=g^{-}\left(\left[\frac{1}{n},+\infty[)\right.\right.$. $\mathcal{V}$ is bounded and contains $\mathbf{y}^{*}$. If $J$ is radial, $\mathcal{V}$ is a closed ball of center $\mathbf{y}^{*}$. We 
denote by $\mathcal{S}=\delta \mathcal{V}$ the frontier of $\mathcal{V}$. As $g$ is continuous, $\mathcal{S}=g^{-}\left(\left\{\frac{1}{n}\right\}\right)$. If $M$ denotes the radius of the ball $\mathcal{V}$, and $\mathrm{I}=\left\{i \in \mathbb{N} / \mathbf{y}_{i} \in \mathcal{V}\right\}, N=\{1, \ldots, n\}$, then:

$$
\begin{aligned}
& \hat{S}_{n}-S_{n}=\sum_{i \in \mathrm{I}} \underbrace{\left(q_{i}-\frac{1}{n}\right)}_{\geq 0} \underbrace{\left\|\mathbf{y}_{i}-\mathbf{y}^{*}\right\|^{2}}_{\leq M}+\sum_{i \in \mathbb{N} \backslash \mathrm{I}} \underbrace{\left(q_{i}-\frac{1}{n}\right)}_{\leq 0} \underbrace{\left\|\mathbf{y}_{i}-\mathbf{y}^{*}\right\|^{2}}_{\geq M} \\
& \hat{S}_{n}-S_{n} \leq M \sum_{i \in \mathbb{N}}\left(q_{i}-\frac{1}{n}\right) \leq 0 .
\end{aligned}
$$

A natural approach consists to examine graphically the behavior of the simulated markov chain which usually takes on a noisy aspect, see for example Fig. 2 One can plot the $\left\{y^{(t)}\right\}$ values w.r.t. $t$. The rapid stabilization of the estimator values indicates that convergence is reached fairly quickly: figure (2,b) shows a fast convergence as soon as the first iterations.

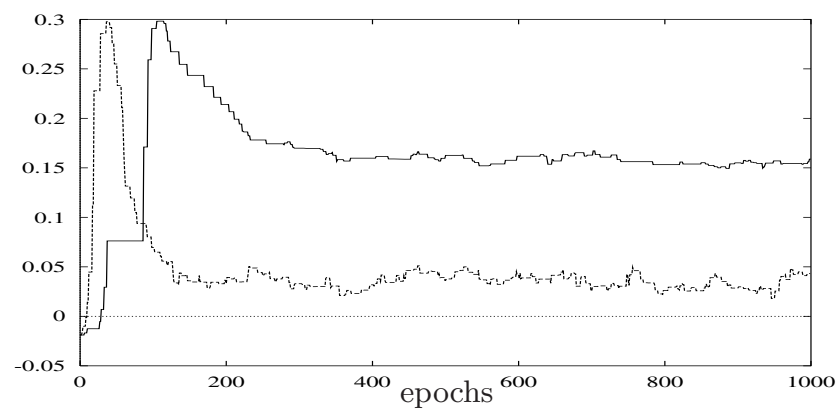

Fig. 2. (a) Markov Chain convergence of the series $\hat{\mathbf{y}}^{(k+1)}=\sum_{k} q\left(\mathbf{x}^{(k)}\right) \mathbf{y}^{(k)} / \sum_{k} q\left(\mathbf{x}^{(k)}\right)$ and $\frac{1}{N} \sum_{i=1}^{N} y^{(i)}$

\section{Simulations}

Example 1 (incomplete data). An interesting example of resolution concerns the (classical) case of mixtures of density. Suppose that $y$ is written as the sum of 4Gaussians and that we do have information only on (any) 3 of them. To what extent, our model is able to recover the fair values of $y$ ? To study this problem, we generate a mixture of 3 Gaussians in the following manner: $x \sim \mathcal{U}_{[0,1]}$ and $y=\sum_{i=1}^{4} p_{i} \phi_{i}(x), \quad \phi_{i} \sim \mathcal{N}\left(\mu_{i}, 1\right)$. At the end of the estimation stage, the numerical values of the parameters are the following $: p_{i}=\{0.1 ; 0.5 ; 0.3 ; 0.12\}$ and $\mu_{i}=\{0.1 ; 0.5 ; 0.7 ;-0.1\}$ respectively.

Now, we must find from the data triplet $\left(\phi_{1}(x), \phi_{2}(x), \phi_{3}(x)\right)$ the value of $y$. At convergence, the plots Fig[3. (a-d) show that the algorithm is capable to rebuild a solution and, moreover, to estimate the sampling from $\phi_{4}(x)$.

Figures 3 . (c-d) demonstrate the differences in scale between log-linear expected/estimated inputs/outputs. Results are quite good for the prediction of $\mathbf{y}$, but 


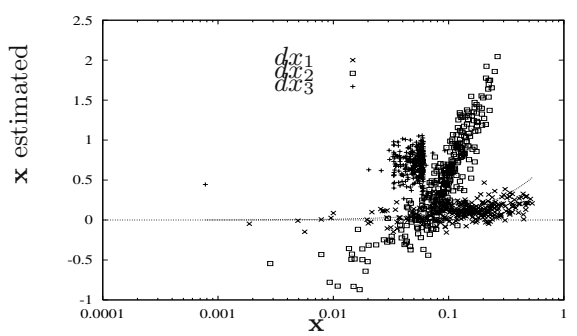

(a)

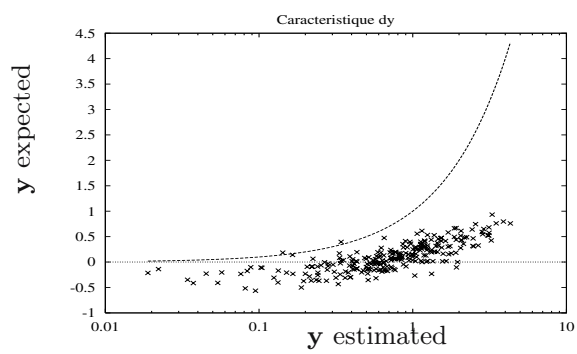

(c)

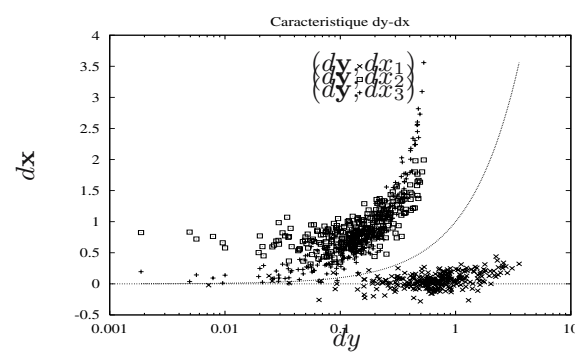

(b)

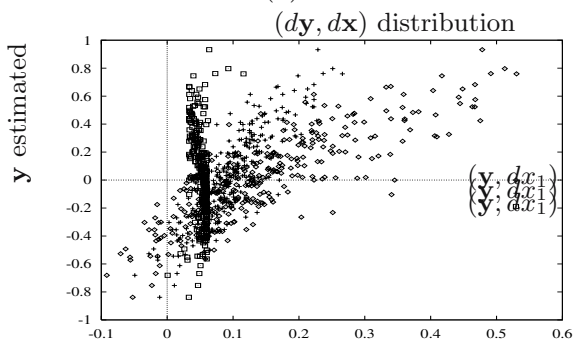

(d)

Fig. 3. Rebuild of a solution in the context of missing data. (a) plot $\left(x_{M_{i}}\left(\mathbf{p}_{i}\right), x_{i}\right)$ (b) plot $(d y, d \mathbf{x})$ (c) plot $d \mathbf{y}(\mathrm{d})$ plot $\left(\mathbf{y}, x_{M_{i}}\left(\mathbf{p}_{i}\right)\right)$. These features are aligned with the diagonal with (few) loss of information caused by the missing variable.

poor for the entries. To counterbalance the loss of information due to the missing model $\phi_{4}$, the algorithm rebuild $\mathbf{y}$ by arranging the distributions $\left(\phi_{i}\right)_{1 \leq i \leq 3}$. This last point is particularly easy to check on the figures 3 . (a-b).

\section{Discussion}

We presented an nonlinear approach capable to solve ill-posed problems:

- that take advantage of the dimensionality (rather than to escape)

- local in the sense that it is effective in a particular area of the signal space.

The inverse model develops an internal representation to the appropriate data structure. By cutting the space signal of its components, the algorithm avoid dimensionality constraints, but must manage as the counterpart, an inference stage that can be risky. It is a parsimonious model, a concept in vogue in the signal processing community. It combines heterogeneous sources, i.e. the information to aggregate do not necessarily come from a single random source; we do not suppose also that the sources are independent. The information provided by a source could have very different forms. In the case of aggregation of information, it is important to identify what are the reliable sources and those in error. There is no question of making the average between the two. A more natural idea is to look for the overlap between these informations in order to emphasize 
areas of consensus. The more these zones are extended, the more the sources are consistent. Conversely, the fact that the sources offer conflicting information suggests that at least one of these sources provides false information. Two coinciding sources should not count for two. In addition, if a source is considered more reliable than another, the information provided by the first have priority over those in the second.

\section{References}

1. Falk, J.E.: Global solutions for signomial programs. Technical Report 6-274, George Washington Univ., Washington DC, USA (1973)

2. Hyvarinen, A., Oja, E.: Independent component analysis: algorithms and applications. Neural Networks 13(4-5), 411-430 (2000)

3. Mendel, J.M.: Maximum-Likelihood deconvolution. A journey into model based signal processing. Springer, New York (1990)

4. Pham, D.T., Cardoso, J.-F.: Independent Component Analysis and Blind Signal Separation (chapter Optimization issues in noisy Gaussian ICA). In: Puntonet, C.G., Prieto, A.G. (eds.) ICA 2004. LNCS, vol. 3195, pp. 41-48. Springer, Heidelberg (2004)

5. Robert, C.P.: Méthodes de simulation en statistiques. Introduction aux méthodes de Monte-Carlo par chînes de Markov. Economica, Paris (1996)

6. Tarantola, A.: Inverse problem theory. Models for data fitting and model parameter estimation. Elsevier, Amsterdam (1987)

7. Tikhonov, A.N., Arsenin, V.Y.: Solutions of ill-posed problems. V.H. Vinsten, Washington (1977) 